
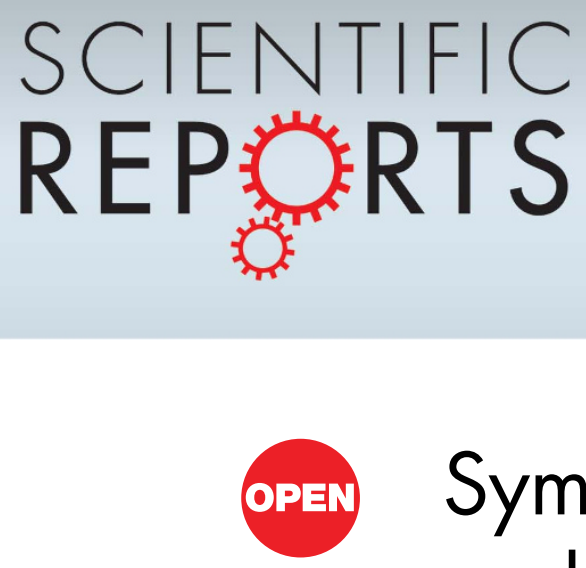

\title{
Symmetry-breaking dynamical pattern and localization observed in the equilibrium vibrational spectrum of $\mathrm{Nal}$
}

SUBJECT AREAS:

MATERIALS PHYSICS

STATISTICAL PHYSICS, THERMODYNAMICS AND NONLINEAR DYNAMICS

CONDENSED MATTER PHYSICS

SPECTROSCOPY

\author{
M. E. Manley' , D. L. Abernathy², N. I. Agladze ${ }^{3}$ \& A. J. Sievers ${ }^{3}$
}

'Lawrence Livermore National Laboratory, Livermore, California 94551, USA, ${ }^{2}$ Oak Ridge National Laboratory, Oak Ridge, Tennessee 37831, USA, ${ }^{3}$ Laboratory of Atomic and Solid State Physics, Cornell University, Ithaca, New York 14853-2501, USA.

Received Intrinsic localized modes (ILMs) - also known as discrete breathers - are localized excitations that form

20 January 2011

Accepted

21 March 2011

Published

14 June 2011

Correspondence and requests for materials should be addressed to M.E.M. (manley3@\|nl.

gov) without structural defects in discrete nonlinear lattices. For crystals in thermal equilibrium ILMs were proposed to form randomly, an idea used to interpret temperature activated signatures of ILMs in $\alpha-\mathrm{U}$ and $\mathrm{NaI}$. Here, however, we report neutron scattering measurements of lattice vibrations in NaI that provide evidence of an underlying organization: (i) with small temperature changes ILMs move as a unit back-and-forth between [111] and [011] orientations, and (ii) when [011] ILMs lock in at $636 \mathrm{~K}$ the transverse optic (TO) mode splits into three modes with symmetry-breaking dynamical structure resembling that of a superlattice, but there are no superlattice Bragg reflections and the pattern itself has crystal momentum. We conclude that this dynamical pattern is not derived from the rearrangement of atoms but from a coherent arrangement of ILMs decorating the crystal lattice in equilibrium.

A lthough ILM formation for driven nonlinear lattices is well established ${ }^{1,2}$, temperature activated ILMs ${ }^{3}$ observed in the $\alpha-\mathrm{U}^{4}$ and $\mathrm{NaI}^{5}$ atomic lattices in thermal equilibrium are not well understood. First, the intensities of ILM signatures (comparable to the normal phonons ${ }^{4,5}$ ) are higher than expected for a dilute concentration. Second, ILM signatures do not follow the expected exponential thermal activation law ${ }^{3}$, but rather appear abruptly at a high temperature followed by modest growth with increasing temperature ${ }^{5,6}$. Third, associated with ILM formation are softened normal phonons positioned at frequencies just below the ILM features ${ }^{4,5}$; coherent modifications to extended modes are not expected for a random distribution of ILMs. Finally, the observed single value of the ILM frequency ${ }^{4,5}$ is unexpected since classical simulations predict a distribution of frequencies ${ }^{7,8}$.

Alternatively, Burlakov ${ }^{9}$ demonstrated theoretically the formation of dynamical patterns for optically driven anharmonic lattices that resemble a train of equal $\mathrm{ILMs}^{10}$. Such dynamical patterns allow for indirect experimental evidence of ILMs based on dynamical breaking of the lattice translational symmetry ${ }^{9,10}$. Similar patterns with ILM constituents were also shown theoretically for the forced-damped Fermi-Pasta-Ulam lattice ${ }^{11}$ and an ILM pattern was predicted for the $\alpha$-U crystal lattice under ion bombardment ${ }^{12}$. For continuous systems driven far from equilibrium, dynamical patterns are often observed ${ }^{13}$ and analogous trains of solitons date back to 1895 with the cnoidal wave trains of Korteweg and De Vries ${ }^{14}$. Unlike with these driven systems, however, here we report ILMs forming a dynamical pattern within the equilibrium thermal vibrations of a crystal. These results could not have been anticipated from previous work on pattern formation in driven systems since such patterns disappear in the absence of the non-equilibrium driving force.

\section{Results}

Figure 1 shows lattice excitation spectra collected near the NaI spectral gap centered near $10 \mathrm{meV}$ for curved sections of $Q-E$ space (momentum-energy). Figure 1a shows data collected for $Q$ directed near the $\langle 111\rangle$ axis for the energy range of interest ( 9 to $11 \mathrm{meV}$ ) at both low $Q_{L}$ and high $Q_{H}$ in similar reciprocal space regions. On increasing temperature from $496 \mathrm{~K}$ to $614 \mathrm{~K}$ a sharp peak develops in the gap near $10.5 \mathrm{meV}$ for both $Q_{L}$ and $Q_{H}$, consistent with the previously reported ILM $^{5}$. On further increasing temperature to $636 \mathrm{~K}$, however, the ILM peak in the gap disappears. The disappearance is particularly clear at $Q_{L}$, where the multiphonon background is smaller ${ }^{15}$, but it is also reproduced in the $Q_{H}$ section. Next, with another small temperature increase to $659 \mathrm{~K}$ the ILM peak reappears in the gap for both $Q_{L}$ and $Q_{H}$ sections. There is also a new feature appearing around $5.8 \mathrm{meV}$ in the acoustic part of the spectrum below the gap, but only in the $Q_{L}$ section. Figure $1 \mathrm{~b}$ shows data collected in a 
a
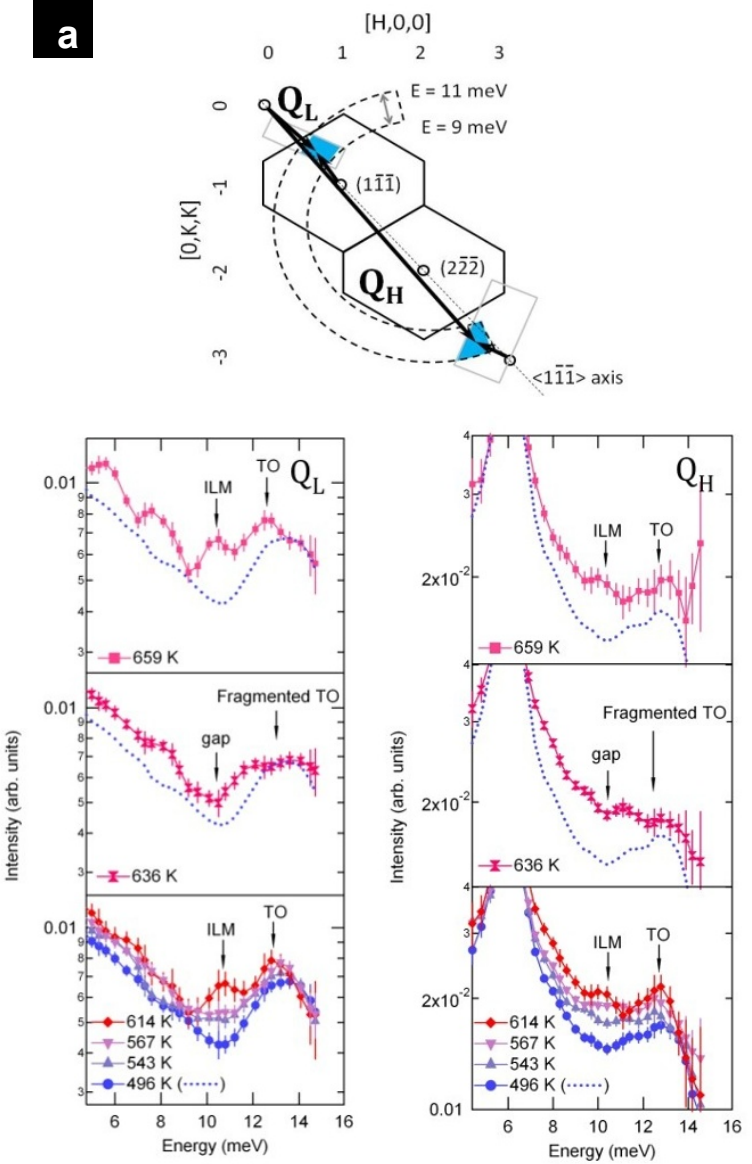
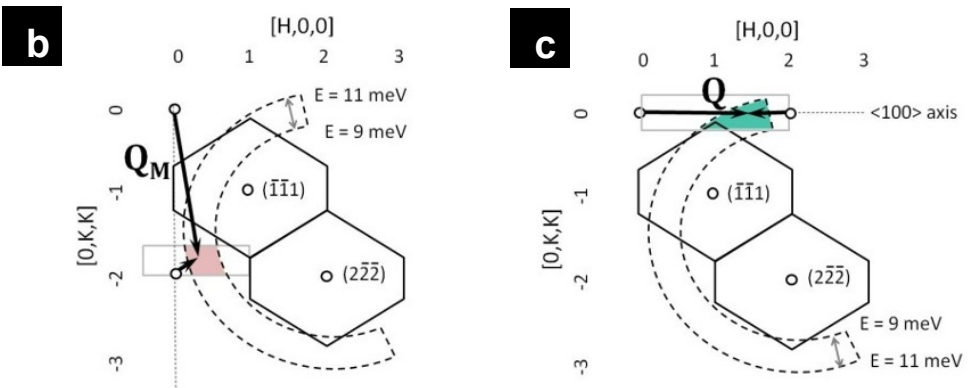

$<0 \overline{1}>$ axis

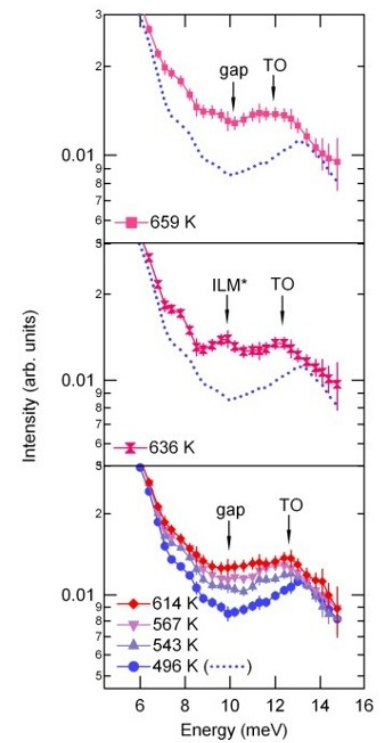

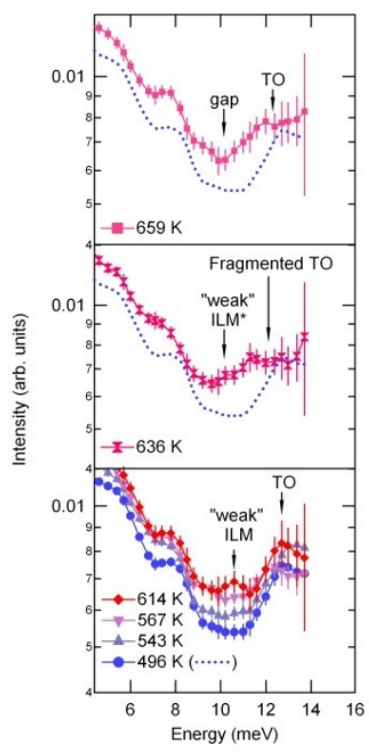

Figure 1 Lattice excitation spectra derived from a curved section of momentum-energy $(Q-E)$ space as a function of temperature. The $Q$ space sampled in these spectra changes with $\boldsymbol{E}$. The curved dashed-line section in the pictures above the data sets shows where the detector banks are projected in the NaI reciprocal lattice for energies between $9 \mathrm{meV}$ and $11 \mathrm{meV}$ (where the ILM feature forms ${ }^{5}$ ). The grey outlined boxes with $\boldsymbol{Q}$ labels indicate the volume of $\boldsymbol{Q}$ space integrated within the plane. Out-of-plane integration for these sections was kept fully open and accounts for about \pm 0.6 reduced lattice units (r. l. u.) in the out of plane $[0, \mathrm{k},-\mathrm{k}]$ direction for energies between 9 and $11 \mathrm{meV}$. (a) Includes low $Q_{L}$ and high $Q_{H}$ sections near the $<111>$ axis just inside the zone. The integration boxes in this case were rotated by $57^{\circ}$ with $Q_{L} 0.2$ by 0.9 r. 1 . u. and $Q_{H} 1$ by $0.5 \mathrm{r}$. l. u. (b) An intermediate $Q_{M}$ region located near the $<011>$ axis; integrating $\mathrm{K}=[-2,-1.7]$ and $\mathrm{H}=[-0.5,1]$. (c) A low $\mathrm{Q}$ region positioned mainly along $<100>$; integrating $\mathrm{K}=[-0.25,0.25]$ and $\mathrm{H}=[0,2]$.

section with $Q$ directed near the $<011>$ axis. These results appear complimentary to those in Fig. 1a. First, from $496 \mathrm{~K}$ to $614 \mathrm{~K}$, as the ILM peak forms in Fig. 1a, no feature appears in the gap in Fig. 1b. But then on heating from $614 \mathrm{~K}$ to $636 \mathrm{~K}$, as the ILM peak in Fig. 1a disappears, a peak appears in the gap at $10 \mathrm{meV}$ labeled ILM*. Finally, at $659 \mathrm{~K}$ as the ILM peak is recovered in Fig. 1a, ILM* disappears in Fig. 1b. Figure 1c shows data in a section along the $<100>$ axis. This appears to be a mixture of what appears in Fig. 1a and $1 \mathrm{~b}$; on heating from $496 \mathrm{~K}$ to $614 \mathrm{~K}$ a weak ILM signature develops in the gap, followed by a weak ILM* signature at $636 \mathrm{~K}$, and very little in the gap at $659 \mathrm{~K}$. Taken together the results of Fig. 1 indicate that the ILMs appearing along $<111>$ at $614 \mathrm{~K}$ collectively reorient into $<011>$ to form the ILM* feature at $636 \mathrm{~K}$ and then move back to $<111>$ at $659 \mathrm{~K}$.

Figure 2 shows $Q-E$ slices taken at $567 \mathrm{~K}$ and $636 \mathrm{~K}$ constructed from multiple scans using MSlice ${ }^{16}$, see methods for details. Figure $2 \mathrm{a}$ shows that ILM*, appearing as a constant energy $10 \mathrm{meV}$ streak in the gap, is dispersionless in $[\mathrm{H},-3,-3]$. Similarly, Figure $2 \mathrm{~b}$ shows that ILM* is also dispersionless in $[-2, \mathrm{~K}, \mathrm{~K}]$. The dispersionless character is consistent with localization and is similar to that observed for the [111] ILM observed previously ${ }^{5}$. Figure $2 c$ shows that along with the appearance of ILM* comes a fragmentation of the TO mode near the $\mathrm{X}$ high symmetry point into at least three features, labeled $\alpha, \beta$, and $\gamma$. This fragmentation can also be seen to extend to other parts of reciprocal space; compare Fig. 2a with Fig. 2 d and Fig. 2 b with Fig. 2 e.
Fragmentation is also evident at $636 \mathrm{~K}$ in Figs. 1a and 1b. No significant changes occur in the longitudinal acoustic (LA) or transverse acoustic (TA) phonon dispersion curves along $[-2, \mathrm{~K}, \mathrm{~K}]$, as can be seen by comparing the curves below $9 \mathrm{meV}$ in Figs. $2 \mathrm{~b}$ and $2 \mathrm{e}$. Figure 3 shows constant $Q$ cuts taken at $636 \mathrm{~K}$ near six X highsymmetry points. For the $(0 \overline{3} \overline{3}) \mathrm{X}$ point (Fig. $3 \mathrm{a})$ the spectrum has strong $\alpha$ and $\gamma$ peaks. However, for the ( $0 \overline{1} \overline{1}) \mathrm{X}$ point (Fig. $3 \mathrm{~b})$ the spectrum contains $\beta$ and $\gamma$, but no $\alpha$ peak. This difference is surprising since $(0 \overline{1} \overline{1})$ and $(0 \overline{3} \overline{3})$ probe the same polarization and are both transverse for the NaI lattice. Next consider Fig. $3 e$, the $(\overline{2} \overline{1} \overline{1}) \mathrm{X}$ point, it is unlike either Fig. 3a or Fig. 3b in that it has $\alpha$ and $\beta$, but no $\gamma$. The $(\overline{1} \overline{2} \overline{2}) \mathrm{X}$ point (Fig. $3 \mathrm{c}$ ) appears similar to $(\overline{2} \overline{1} \overline{1})$ with $\alpha$ and $\beta$ peaks in the spectrum. The $(\overline{3} \overline{2} \overline{2}) \mathrm{X}$ point (Fig. $3 \mathrm{f}$ ) appears to have no clear TO features. Finally, the $(\overline{2} \overline{3} \overline{3}) \mathrm{X}$ point (Fig. $3 \mathrm{~d}$ ) contains mostly $\alpha$ and $\gamma$ with a weak $\beta$ peak. Following the ILM* peak intensity going from Figs. $3 \mathrm{c}$ to $3 \mathrm{~d}$ to $3 \mathrm{f}$ and to $3 \mathrm{e}$ shows that its intensity decreases and finally vanishes as the orientation moves away from [011] towards [100]. This is consistent with the [011] orientation deduced for ILM* from Fig. 1. These results show that with the appearance of the [011] ILM*s the TO splits into three modes with symmetry-breaking coherent structure.

Figure 4 provides a map describing how the TO mode fragments change between the $\mathrm{X}$ points across Brillioun Zones. The $[\mathrm{H},-3,-3]$ $Q$ slice in Fig. 4a shows that $\gamma$ is absent in a section from about $\mathrm{H}=-(1+2 / 3)$ to the zone center at $\mathrm{H}=-1$. Similarly, the $[H,-1,-1]$ 
a

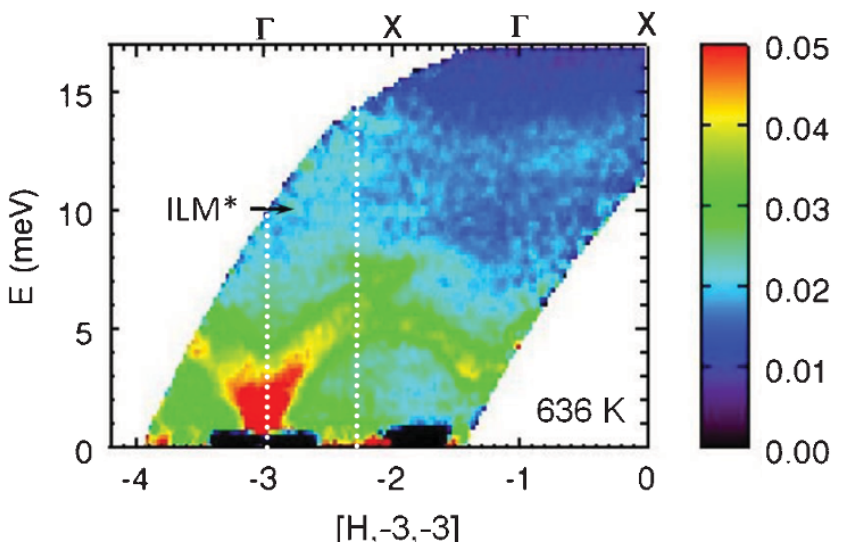

b

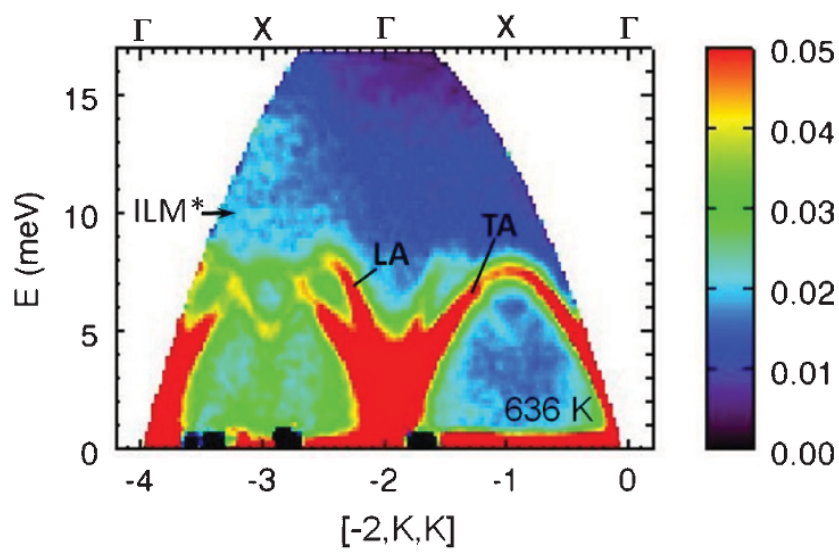

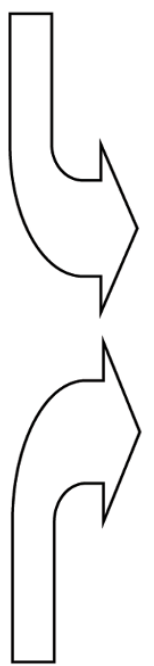

c

C $\mathrm{K}=[-3.2,-2.8] \mathrm{H}=[-2.5,-1,8] \mathrm{kmk}=[-0.25,0.25]$

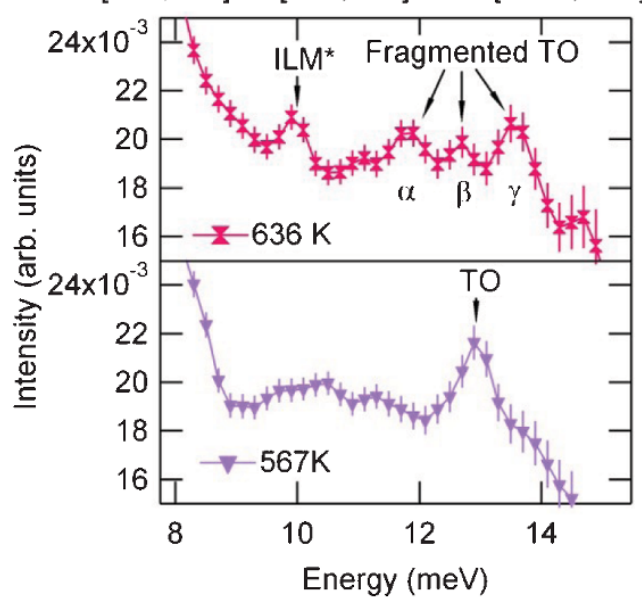

\section{$[0, K, K]$}

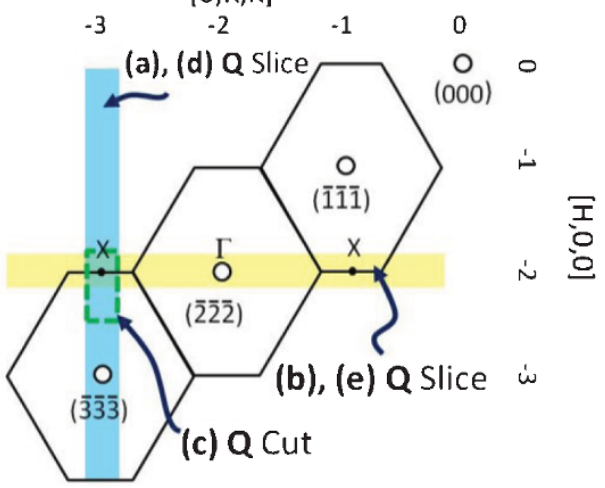

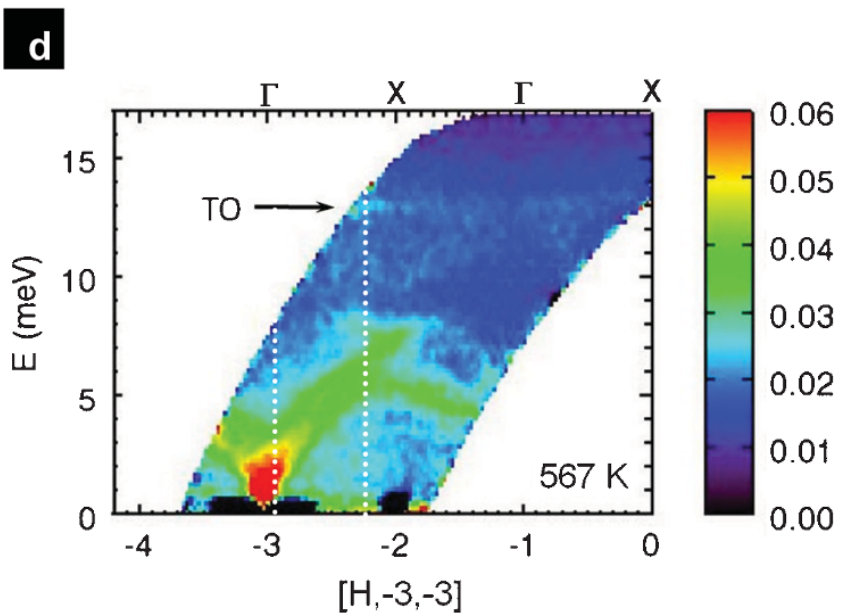

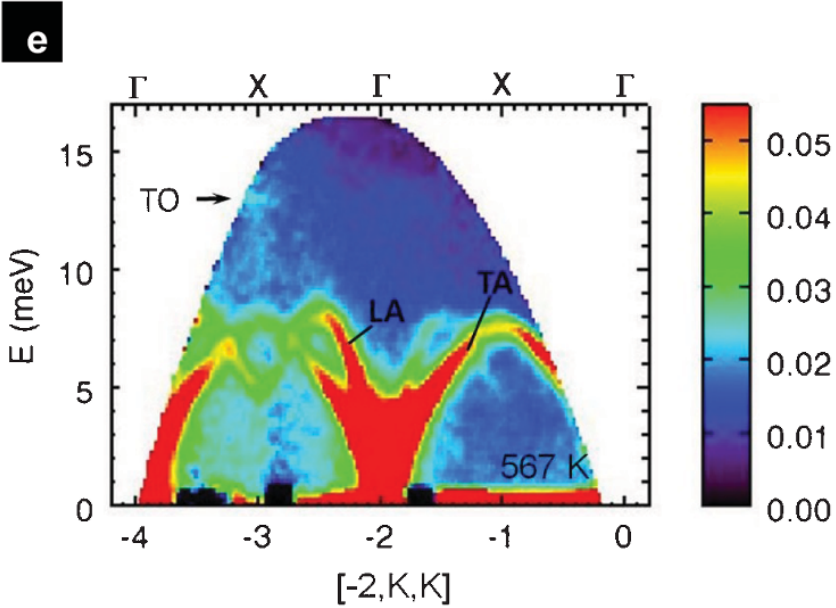

Figure $2 \mid$ Phonon dispersion $Q-E$ slices along $[\mathrm{H},-3,-3]$ and $[-2, \mathrm{~K}, \mathrm{~K}]$ along with a $Q$ cut. All slices and the cut section are indicated schematically in the image at right center. The out of plane $[0, \mathrm{k},-\mathrm{k}]$ direction is constrained to $\mathrm{k}=[-0.25,0.25]$ for all panels. (a) Slice along $[\mathrm{H},-3,-3]$ with the $[0, \mathrm{~K}, \mathrm{~K}]$ constrained to $\mathrm{K}=[-3.2,-2.8]$ at $636 \mathrm{~K}$. (b) Slice along $[-2, \mathrm{~K}, \mathrm{~K}]$ with $[\mathrm{H}, 0,0]$ constrained to $\mathrm{H}=[-2.2,-1.8]$ at $636 \mathrm{~K}$. (c) Cuts taken within the white dotted line section of (a) indicated by arrow at both $636 \mathrm{~K}$ and $567 \mathrm{~K}$. The range for $\mathrm{K}$ along [0,K,K], $\mathrm{H}$ along $[\mathrm{H}, 0,0]$, and k along [0,k,-k] (designated kmk for k minus $\mathrm{k}$ ) are indicated at the top of the panel. Slices (d) and (c) are the same as (a) and (b) respectively, except at $567 \mathrm{~K}$.

$Q$ slice in Fig. $4 b$ shows $\alpha$ is absent in a section from $H=-1 / 3$ to $\mathrm{H}=+1 / 3$. The $[H,-1,-1] Q$ slice in Fig. $4 c$ shows a larger section without the $\gamma$ feature that extends from $\mathrm{H}=-0.5$ to $\mathrm{H}=-2$. The $[-1, \mathrm{~K}, \mathrm{~K}] Q$ slices in Figs. $4 \mathrm{~d}$ and $4 \mathrm{e}$ show that the $\alpha$ and $\beta$ features appear in narrow ranges along this direction near the $\Gamma$ points at
$K=-3$ and $K=-1$. In Fig. $4 \mathrm{e} \alpha$ and $\beta$ reemerge near $K=-(1+1 / 3)$. Additional weaker features at $11 \mathrm{meV}$ appear near both of these $\Gamma$ points, highlighted with white dashed ovals. Finally, the $[-2, \mathrm{~K}, \mathrm{~K}] \boldsymbol{Q}$ slice in Fig. $4 \mathrm{f}$ shows a small region where $\operatorname{ILM}^{*}, \alpha, \beta$, and $\gamma$ all appear simultaneously near an $\mathrm{X}$ point. 

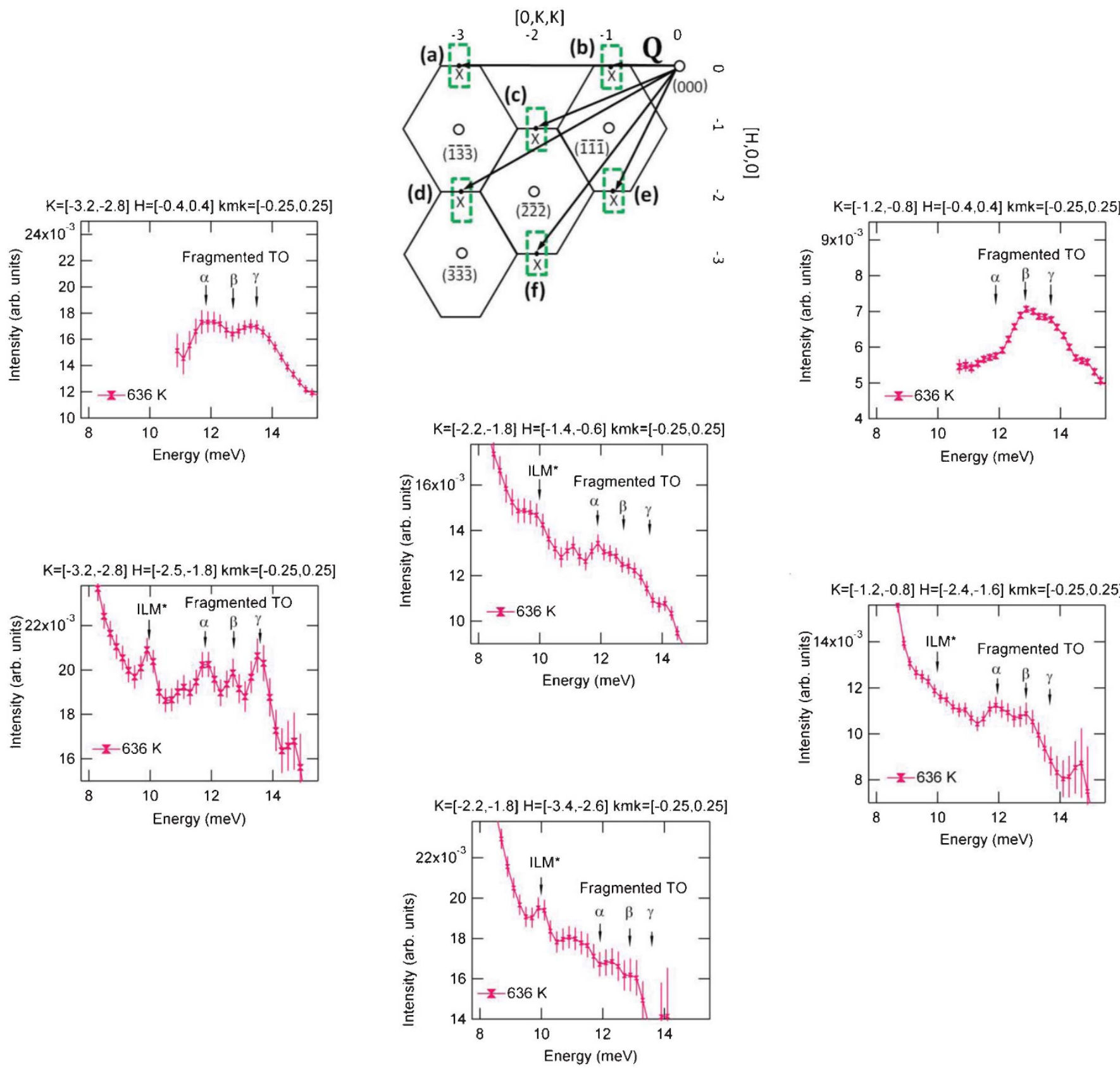

Figure $3 \mid$ A series of $Q$ cuts at six $\mathrm{X}$ high-symmetry points. The range for $\mathrm{K}$ along [0,K,K], $\mathrm{H}$ along [H,0,0], and $\mathrm{k}$ along [0,k,-k] (designated $\mathrm{kmk}$ for $\mathrm{k}$ minus k) are indicated at the top of each panel. The six X points are: (a) $(0 \overline{3} \overline{3}),(\mathrm{b})(0 \overline{1} \overline{1}),(\mathrm{c})(\overline{1} \overline{2} \overline{2}),(\mathrm{d})(\overline{2} \overline{3} \overline{3}),(\mathrm{e})(\overline{2} \overline{1} \overline{1})$, and (f) $(\overline{3} \overline{2} \overline{2})$. All of these cuts are indicated in the drawing at top center.

\section{Discussion}

The TO mode splitting and discontinuous sectioning at fractions of a zone length are similar to zone folding effects seen with superlattices ${ }^{17}$, but here there are no corresponding superlattice Bragg reflections ( $i n$ situ diffraction was monitored using $\boldsymbol{Q}$ slices near the elastic energy and showed no crystal structure changes through all measurements, as expected). Figure 5a shows how equally spaced ILM-like bond-defect modes in a $1 \mathrm{D}$ model give rise to dynamical superlattice properties without a structural superlattice. The $2 / 3$ sections along $[\mathrm{H}, 0,0]$ in $\mathrm{NaI}$ (Fig. 4) suggest a tripling of the unit cell along [100]. This allows for threefold splitting of the [100] TO mode into $\alpha, \beta$, and $\gamma$, while ILM*s originate along $[011]$ and $[0,-1,1]$ but appear at propagation wave vectors with components along [100] owing to localization. Figure $5 \mathrm{~b}$ shows that this superlattice is commensurate with the observed $2 / 3$ sections of the split TO mode, but that contrary to lattice theory the discontinuities in the modes all occur at zone centers rather than at zone boundaries, including the $(\overline{1} \overline{3} \overline{3})$ center common to all NaI superlattices. Noting that discontinuities must occur at zone boundaries suggests that the dynamical superlattice is offset in momentum space by $[ \pm 1 / 3,0,0]$, implying that the dynamical superlattice itself has crystal momentum.

These observations are surprising for a crystal in thermal equilibrium. From equipartition the amplitude of the gap feature is one tenth that calculated for the 3-D NaI ILM model ${ }^{7}$ and driven 1-D and 2-D models require sufficient carrier amplitude to initiate pattern forming modulation instabilities ${ }^{9}$. Measurements on material from a third source containing an impurity gap mode present at all temperatures (not shown) showed a slightly broader-weaker ILM feature form at high temperatures. Our conclusion is that these patterns form easier with temperature than classical models suggest, and 
a

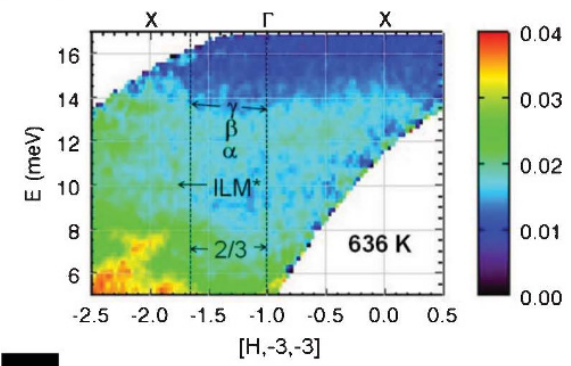

d

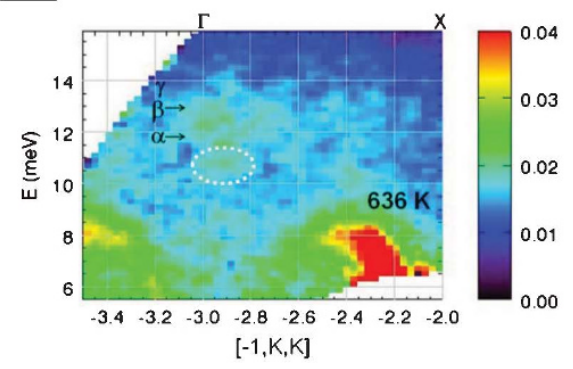

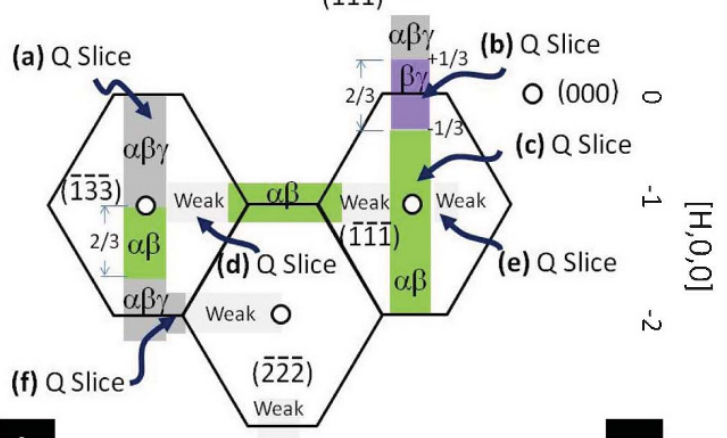

b

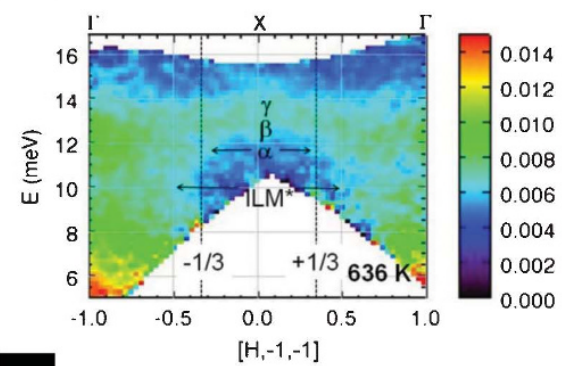

e

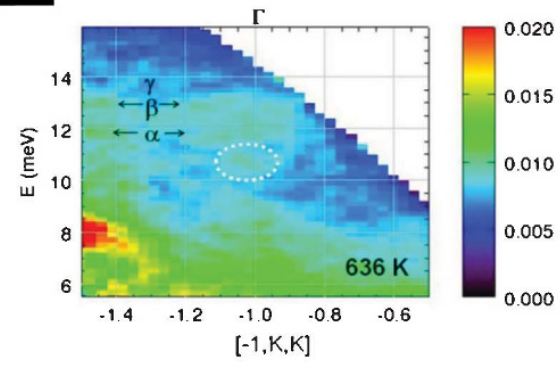

C

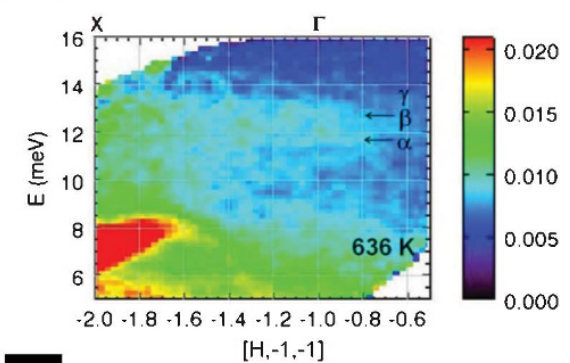

f

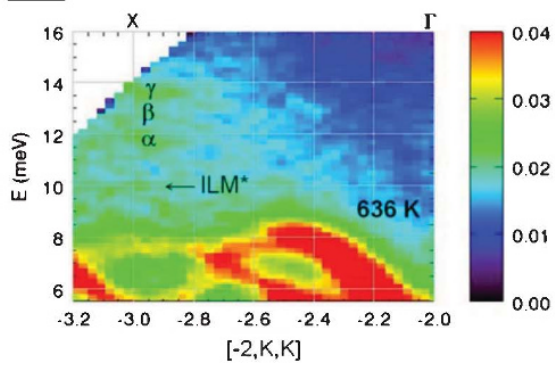

Figure $4 \mid$ Map of the $\alpha, \boldsymbol{\beta}$, and $\gamma$ TO fragment coherent structure. Regions with just $\beta$ and $\gamma$ intensity are shaded purple, regions with just $\alpha$ and $\beta$ intensity are shaded green, and regions with all three are shaded grey. Location where all three were not clearly visible are designated "weak". For all slices the out-of-plane $[0, \mathrm{k},-\mathrm{k}]$ was constrained to $\mathrm{k}=[-0.25,0.25]$. (a) Shows a slice along $[\mathrm{H},-3,-3]$ where a segment of $\gamma$ is absent in a 2/3 r. 1 . u. section. (b) Shows a slice along $[H,-1,-1]$ around $H=0$ where a segment of $\alpha$ is absent is absent in a $2 / 3 \mathrm{r}$. 1 . u. section from $H= \pm 1 / 3$. (c) Shows a slice along $[H,-1,-1]$ around $\mathrm{H}=1.5$ where a larger section with $\gamma$ absent is observed. (d) Shows a slice along $[-1, \mathrm{~K}, \mathrm{~K}]$ around $\mathrm{K}=-3$ where $\alpha$ and $\beta$ are observed in a narrow section. An additional feature is indicated in the white dashed oval near $11 \mathrm{meV}$. (e) Shows a slice along $[-1, \mathrm{~K}, \mathrm{~K}]$ around $\mathrm{K}=-1$ that appears similar to the $\mathrm{K}=-3$ point, including the extra feature near $11 \mathrm{meV}$. (f) Shows a slice along $[-2, \mathrm{~K}, \mathrm{~K}]$ where all primary features $\alpha, \beta, \gamma$, and ILM* are visible in a region near $\mathrm{K}=-3$.

depend somewhat on crystal quality. The observed single-energy ILM peak ${ }^{4,5}$ is expected for a dynamical pattern built from equal ILMs $^{9,10}$. The abrupt appearance of the ILM at high temperatures followed by modest growth with increasing temperatures ${ }^{4,5}$ is consistent with a pattern of ILMs. The collective changes of ILMs between [111] and [011] orientations (Fig. 1) are expected for patterns of ILMs transitioning between configurations. Finally, the observed symmetry-breaking dynamical pattern (Figs. 2-4) requires coherent alterations to interatomic force constants without changing atomic arrangements, the hallmark of a dynamical pattern built from ILMs. Unlike with a random distribution of ILMs, which could be stabilized by configurational entropy ${ }^{3}$, this dynamical pattern of ILMs poses a theoretical challenge as to how it is achieved in thermal equilibrium. While simulations have shown the existence of ILMs within the thermal-equilibrium vibrational spectrum of a $2 \mathrm{D}$ nonlinear lattice ${ }^{19}$, ILM order in equilibrium has not been reported. However, for the conceptually similar problem of substituting atoms of a different species into a crystal, ordering transitions only occur for narrow near-stoichiometric concentrations ${ }^{20}$. By analogy, the narrow temperature range of the ILM pattern may correspond to a temperature where the ILM concentration is a rational fraction of the lattice sites, allowing for a well ordered low-energy arrangement. A limitation of this analogy is that the ILM concentration need not obey the same conservation laws as atomic substitution. Simulations of the ILMs in $\mathrm{NaI}^{7}$ show that ILMs are accompanied by local strains, including both tensile and compressive fields. A well-ordered arrangement of ILMs that overlap the compressive and tensile strain fields of adjacent ILMs would lower the strain energy, providing a possible driving force for order.

\section{Methods}

Time-of-flight inelastic neutron scattering spectra were obtained on a large single crystal of $\mathrm{NaI}(0.002 \mathrm{Tl})$ (same source as crystal but different from the pure NaI powder used in Ref. [5]) mounted on a rotating furnace using the wide Angular-Range Chopper Spectrometer (ARCS) at the Spallation Neutron Source (SNS) of Oak Ridge National Laboratory. Empty can runs were collected at measurement temperatures and were subtracted from all data sets. All measurements were taken with (HKK) in the scattering plane. In the first set of measurements curved sections of $Q-E$ space (momentum-energy) were measured with the crystal held at a single angle at temperatures near the reported ILM formation temperature ( $555 \mathrm{~K}^{5}$, although a subsequent calibration of the furnace at NIST indicates it was closer to $575 \mathrm{~K}$ ): $496 \mathrm{~K}$, $543 \mathrm{~K}, 567 \mathrm{~K}, 614 \mathrm{~K}, 636 \mathrm{~K}$, and $659 \mathrm{~K}$. The orientation was such that the [100] axis 
a

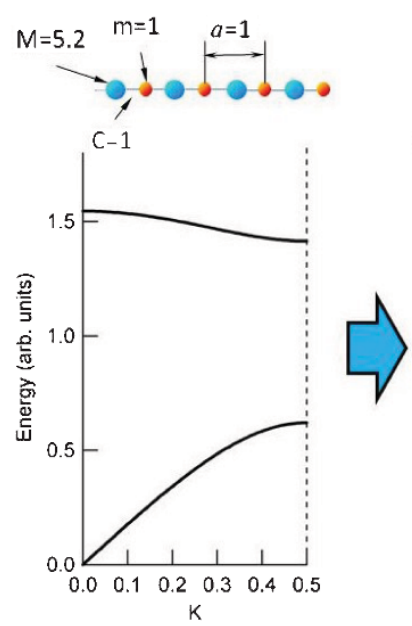

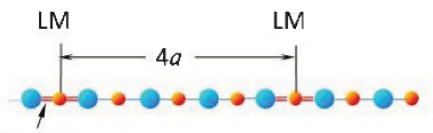

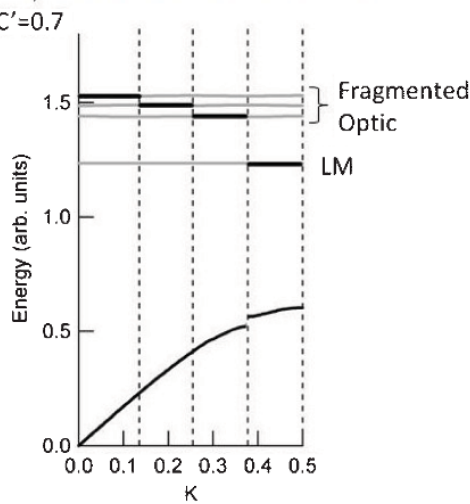

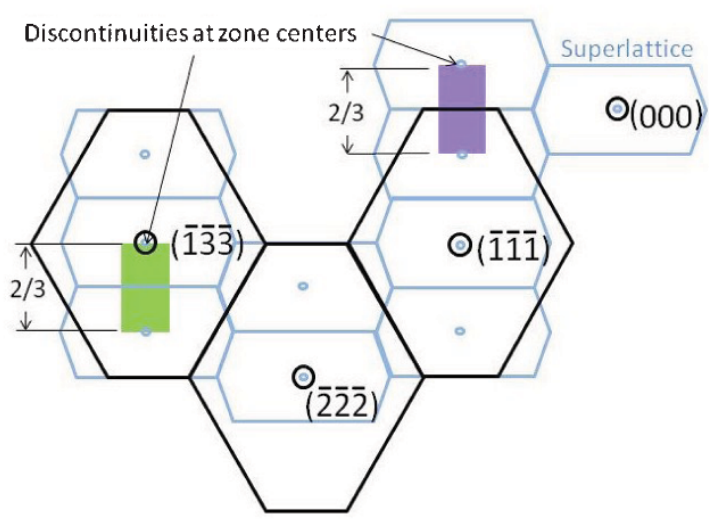

Figure $5 \mid$ Dynamical superlattices formed from ordered arrangements of ILM-like local modes. (a) One dimensional diatomic lattice dynamical model after Kittel ${ }^{18}$ with nearest neighbor force constants $\mathrm{C}=1$ and a mass ratio $\mathrm{M} / \mathrm{m}=5.2$, with and without ILM-like local modes. An array of ILM-like local modes is introduced by reducing the force constants on every fourth light atom to $C^{\prime}=0.7$, simulating the local softening expected with ILMs. The resulting phonon dispersion curves, unfolded to the same K-space scale, show that the single optic mode is fragmented into three closely spaced segments plus the local mode dropping down further into the gap. The acoustic mode shows minimal splitting by comparison. (b) Superlattice zone scheme in the $[\mathrm{HKK}]$ plane for $\mathrm{NaI}$ with the unit cell tripled along [100] (blue lines) projected on the parent zone scheme (black lines). The purple and green sections reproduce the experimental discontinuities in the TO fragments from Fig. 4.

of the crystal was at $39.7^{\circ}$ with respect to the incident beam, and was chosen so that nearly equivalent zone sections along the [111] direction were aligned simultaneously with the high and low angle detector regions at the energy of interest $(10 \mathrm{meV})$, providing a built in consistency check. In the second set of measurements comprehensive 4-dimensional $Q-E$ volumes of data where obtained at $567 \mathrm{~K}$, and $636 \mathrm{~K}$, by rotating the angle between [100] and the incident beam in 1 degree steps, collecting a scan at each angle, and stitching the data together using the MSlice software package in DAVE ${ }^{16}$. The angles ranged from $70^{\circ}$ to $100^{\circ}$ for $567 \mathrm{~K}$, and between $65^{\circ}$ and $105^{\circ}$ for $636 \mathrm{~K}$.

1. Campbell, D. K., Flach, S. \& Kivshar, Y. S. Localizing energy through nonlinearity and discreteness. Physics Today 57, 43-49 (2004).

2. Flach, S. \& Gorbach, A. Discrete breathers-Advances in theory and applications. Phys Reports 467, 1-116 (2008).

3. Sievers, A. J. \& Takeno, S. Intrinsic localized modes in anharmonic crystals. Phys. Rev. Lett. 61, 970 (1988).

4. Manley, M. E., Yethiraj, M., Sinn, H., Volz, H. M., Alatas, A., Lashley, J. C., Hults, W. L., Lander, G. H., \& Smith, J. L. Formation of a new dynamical mode in $\alpha$-uranium observed by inelastic neutron and x-ray scattering. Phys. Rev. Lett. 96, 125501 (2006)

5. Manley, M. E., Sievers, A. J., Lynn, J. W., Kiselev, S. A., Agladze, N. I., Chen, Y., Llobet, A., \& Alatas, A. Intrinsic localized modes observed in the hightemperature vibrational spectrum of NaI. Phys. Rev. B 79, 134304 (2009).

6. Manley, M. E., Alatas, A., Trouw, F., Leu, B. M., Lynn, J. W., Chen, Y. \& Hults, W. L. Intrinsic nature of thermally activated dynamical modes in $\alpha$-U: Nonequilibrium mode creation by x-ray and neutron scattering. Phys. Rev. B 77, 214305 (2008).

7. Kiselev, S. A., \& Sievers, A. J. Generation of intrinsic vibrational gap modes in three-dimensional ionic crystals. Phys. Rev. B 55, 5755 (1997).

8. Khadeeva, L. Z., \& Dimitriev, S. V. Discrete breathers in crystals with $\mathrm{NaCl}$ structure. Phys. Rev. B 81, 214306 (2010).

9. Burlakov, V. M. Interference of mode instabilities and pattern formation in anharmonic lattices. Phys. Rev. Lett. 80, 3988 (1998).

10. Burlakov, V. M. Spatial- and spatio-temporal pattern formation in optically driven discrete systems. Int. J. of Mod. Phys. B 13, Issue 07, pp. 791-805 (1999).

11. Khomeriki, R., Lepri, S., \& Ruffo, S. Pattern formation and localization in the forced-damped Fermi-Pasta-Ulam lattice. Phys. Rev. E 64, 056606 (2001).

12. Dubovsky, O. A., Orlov, A. V. Emission of supersonic soliton wave beamsgenerators of restructuring of nanocrystals under atom bombardment, and the self-organization of a dynamical superlattice of complexes of soliton atomic vibrations. Phys. of Solid State 52, 5, 899-903 (2010).

13. Cross, M. C., Hohenberg, P. C. Pattern formation outside of equilibrium. Rev. Mod. Phys. 65, 851 (1993).
14. Korteweg, D. J. \& de Vries, G. On the change of form of long waves advancing in a rectangular canal, and on a new type of long stationary waves. Phil. Mag. 39:240, 422-443 (1895)

15. Squires, G. L. Introduction to the Theory Neutron Scattering (Dover, New York, 1978) p. 61.

16. Azuah, R.T., Kneller, L.R., Qiu, Y., Tregenna-Piggott, P.L.W., Brown, C.M., Copley, J.R.D., \& Dimeo, R.M. DAVE: A Comprehensive Software Suite for the Reduction, Visualization, and Analysis of Low Energy Neutron Spectroscopic Data.J. Res. Natl. Inst. Stan. Technol. 114, 341 (2009).

17. Sapriel, J., Djafari Rouhani, B. Vibrations in superlattices. Surf. Sci. Rep. 10, 189275 (1989).

18. Kittel, C., Introduction to Solid State Physics $7^{\text {th }}$ Edition (John Wiley \& Sons, New York, 1996) p. 104-107.

19. Eleftheriou, M., Flach, S. Discrete breathers in thermal equilibrium: distributions and energy gaps. Physica D 202, 142-154 (2005).

20. Porter, D. A., Easterling, K. E., Phase Transformations in Metals and Alloys $2^{\text {nd }}$ Edition (Chapman \& Hall, London, 1993) p. 24.

\section{Acknowledgements}

Work was partly performed under the auspices of the U.S. Department of Energy by Lawrence Livermore National Laboratory under Contract No. DE-AC52-07NA27344. Research at Oak Ridge National Laboratory's Spallation Neutron Source was sponsored by the Scientific User Facilities Division, Office of Basic Energy Sciences, U.S. Department of Energy. The Cornell effort was supported by NSF grant DMR-0906491. M.E.M acknowledges helpful correspondence with P. S. Riseborough. A. J. S. thanks J. B. Page, J. P. Sethna, and V. M. Burlakov for insightful comments.

\section{Author contributions}

Scattering experiments were performed by D.L.A., M.E.M., and N.I.A. The data was analyzed and interpreted by M.E.M, D.L.A and A. J. S. The crystals were supplied by N.I.A and A.J.S. The paper was written by M.E.M.

\section{Additional information}

Competing interests statement: The authors declare that they have no competing financial interests.

License: This work is licensed under a Creative Commons

Attribution-NonCommercial-ShareAlike 3.0 Unported License. To view a copy of this license, visit http://creativecommons.org/licenses/by-nc-sa/3.0/

How to cite this article: Manley, M. E., Abernathy, D. L., Agladze, N. I. \& Sievers, A. J. Symmetry-breaking dynamical pattern and localization observed in the equilibrium vibrational spectrum of NaI. Sci. Rep. 1, 4; DOI: 10.1038/srep00004 (2011). 